\title{
Existence of Traveling Waves for Ratio-dependent Predator-prey System with Nonlocal Diffusion
}

\author{
Ke Li, Hongmei Cheng* \\ School of Mathematics and Statistics, Shandong Normal University, Jinan, P.R. China
}

Email address:

hmcheng@sdnu.edu.cn (Hongmei Cheng)

${ }^{*}$ Corresponding author

\section{To cite this article:}

$\mathrm{Ke} \mathrm{Li}$, Hongmei Cheng. Existence of Traveling Waves for Ratio-dependent Predator-prey System with Nonlocal Diffusion. American Journal of Applied Mathematics. Special Issue: Application of Nonlinear Analysis. Vol. 8, No. 5, 2020, pp. 236-246. doi: 10.11648/j.ajam.20200805.11

Received: February 18, 2020; Accepted: August 14, 2020; Published: August 25, 2020

\begin{abstract}
In this paper, we study the traveling waves for the ratio-dependent predator-prey model with nonlocal diffusion, which is devoted to the existence and nonexistence of traveling wave solution. This model incorporates the ratio-dependent functional response into the Lotka-Volterra type system, and both species obey the logistic growth. Firstly, we construct a nice pair of upper and lower solutions when the wave speed is greater than the minimal wave speed. Then by applying Schauder's fixed point theorem with the help of suitable upper and lower solutions, we can obtain the existence of traveling waves when the wave speed is greater than the minimal wave speed. Moreover, in order to prove the limit behavior of the traveling waves at infinity, we construct a sequence that converges to the coexistence state. Finally, by using the comparison principle, we obtain the nonexistence of the traveling waves when the wave speed is greater than 0 and less than the minimal wave speed. The difficulty of this paper is to construct a suitable upper and lower solution, which is also the novelty of this paper. Under certain restricted condition, this paper concludes the existence and the nonexistence of the traveling waves for the ratio-dependent predator-prey model with nonlocal diffusion.
\end{abstract}

Keywords: Traveling Wave Solution, Predator-prey Model, Nonlocal Diffusion, Ratio-dependent Functional Response, Schauder's Fixed Point Theorem, Comparison Principle

\section{Introduction}

The interaction between the predator and the prey constitutes a dynamic relationship that has been one of the main topics in ecological research, which is important for studying the distribution of organisms and the balance of the environment.

This relationship can be described by the dynamic behavior of some mathematical models. To describe the predator-prey model, Tanner in [28] considered the following ordinary differential system

$$
\left\{\begin{array}{l}
\frac{d u(t)}{d t}=u(t)[1-u(t)]-\Pi(u(t)) v(t), \\
\frac{d v(t)}{d t}=r v(t)\left(1-\frac{v(t)}{u(t)}\right)
\end{array}\right.
$$

where $\Pi(u)=\frac{m u}{A+u}(m>0, A>0)$ denotes the functional response to predation suggested by Holling in [15] and $r$ denotes the growth rate of predator. The second equation of (1) means that the intrinsic population growth rate $r$ affects not only the potential increase of the population but also its decrease. The classical Lotka-Volterra model and its modified models have been studied for the stability of equilibria and the existence of traveling waves, see [8, 14, 17, 19, 29, 30].

By taking into account the effect of the diffusion, reactiondiffusion predator-prey models have been established to describe the invasion of a predator species [11, 12, 22, 26]. Some previous work for dynamics of diffusive HollingTanner predator-prey systems on a bounded region can be found in [3, 25]. Zuo and Shi in [31] has studied the reactiondiffusion Holling-Tanner type predator-prey system with ratio-dependent functional response

$$
\left\{\begin{array}{l}
u_{t}(x, t)=d u_{x x}(x, t)+u(x, t)(1-u(x, t))-\frac{A u(x, t) v(x, t)}{u(x, t)+a v(x, t)}, \\
v_{t}(x, t)=v_{x x}(x, t)+B v(x, t)\left(1-\frac{v(x, y)}{u(x, t)}\right)
\end{array}\right.
$$


the two species move randomly along a one-dimensional region $\mathrm{R}$, and the parameter $d>0$ is a rescaled diffusion coefficient of the prey species while the diffusion coefficient for the predator is rescaled to be 1 . They established the existence of traveling wave solution by using the upper and lower solutions method and proved the existence of periodic traveling wave train by using the Hopf bifurcation theorem. Upper-lower solution and Fixed point theory have also be used to prove the existence of traveling wave solutions in a quasimonotone system, see $[13,16,20,21]$.

About the discrete diffusive ratio-dependent predator-prey model, Zhang and Su in [27] considered the following system

$$
\left\{\begin{array}{l}
\frac{d u_{j}(t)}{d t}=\mathcal{D}\left[u_{j}(t)\right]+u_{j}(t)\left(1-u_{j}(t)\right)-\frac{A u_{j}(t) v_{j}(t)}{u_{j}(t)+a v_{j}(t)} \\
\frac{d v_{j}(t)}{d t}=d \mathcal{D}\left[v_{j}(t)\right]+\beta v_{j}(t)\left(1-\frac{v_{j}(t)}{u_{j}(t)}\right)
\end{array}\right.
$$

where $j \in \mathbb{Z}, t>0, d>0, \mathcal{D}\left[z_{j}\right]=z_{j+1}+z_{j-1}-2 z_{j}$ and $A$,

$$
J * u(x, t)=\int_{\mathbb{R}} J(y) u(x-y, t) d y, J * v(x, t)=\int_{\mathbb{R}} J(y) v(x-y, t) d y .
$$

$(J * u-u)(x, t)$ and $(J * v-v)(x, t)$ represent nonlocal diffusion processes $[1,10,23,24]$. Meanwhile, many researchers study the properties of the traveling wave solution for the reaction-diffusion systems with nonlocal $a, \beta$ are positive constants. Zhang and $\mathrm{Su}$ obtained the existence of invasion traveling wave solution of (2) by applying Schauder's fixed point theorem with the help of suitable upper and lower solutions.

Considering of special diffusion, the standard Laplacian operator is corresponding to expected values for individuals moving under a Brownian process. But the movement of individuals is free and random which can not be limited in a small area. So various integral operators have been widely applied to describe the nonlocal diffusion. The nonlocal reaction-diffusion system takes the form

$$
\left\{\begin{array}{l}
\frac{\partial u(x, t)}{\partial t}=d_{1}(J * u(x, t)-u(x, t))+f(u(x, t), v(x, t)), \\
\frac{\partial v(x, t)}{\partial t}=d_{2}(J * v(x, t)-v(x, t))+g(u(x, t), v(x, t)),
\end{array}\right.
$$

where $J(x)$ is the diffusion kernel given by

$$
\left\{\begin{array}{l}
\frac{\partial u(x, t)}{\partial t}=d_{1}(J * u(x, t)-u(x, t))+u(x, t)(1-u(x, t))-\frac{\beta u(x, t) v(x, t)}{u(x, t)+a v(x, t)}, \\
\left.\frac{\partial v(x, t)}{\partial t}=d_{2} J * v(x, t)-v(x, t)\right)+r v(x, t)\left(1-\frac{v(x, t)}{u(x, t)}\right)
\end{array}\right.
$$

where $u(x, t)$ and $v(x, t)$ are the population densities of the prey and predator species at the location $x$ and time $t$ respectively, $J * u(x, t)$ and $J * v(x, t)$ are the same as the previous (3); the parameters $\beta, a, d_{i}(i=1,2)$ and $r$ are positive constants. The parameters $d_{i}(i=1,2)$ are diffusion rates for the prey and predator individuals, respectively, $r$ is the intrinsic growth rate of predator, $\beta$ is the capturing rate, and $a$ is the half-capturing saturation constant. If $\beta=0$, then the first equation of system (4) is simplified to fisher's KPP equation. If the preys are only as food for the predator, that is, $u \equiv 1$, then the second equation of system (4) is simplified to fisher's KPP equation. Some authors obtained the nonexistence of traveling wave solutions of predator systems by considering the related Cauchy problem of fisher's equation, see [31]. Throughout this paper, we need the below assumptions of the kernel function $J$.

Assumption $1.1\left(K_{1}\right)$ The function $J$ is a smooth function in $\mathbb{R}$ and satisfies $J \in C^{1}(\mathbb{R}), J(y)=J(-y) \geq 0$, $\int_{\mathbb{R}} J(y) d y=1$.

$\left(K_{2}\right)$ There exists $\lambda_{0} \in(0,+\infty]$ such that $\int_{\mathbb{R}} J(y) e^{-\lambda y} d y<+\infty \quad$ for any $\lambda \in\left[0, \lambda_{0}\right) \quad$, and $\int_{\mathbb{R}} J(y) e^{-\lambda y} d y \rightarrow+\infty$ as $\lambda \rightarrow \lambda_{0}-0$.

In this work, we mainly study the existence of the traveling wave solution which connects the predator free state $(1,0)$ with the coexistence state $(k, k)$ of the system (4), where $k=1-\frac{\beta}{a+1}>0$, when $0<\beta<a$. We will obtain that there exists $c^{*}>0$ such that for $c>c^{*}$, the system (4) admits traveling wave solution with wave speed $c$; for $0<c<c^{*}$, the system (4) has no invasion traveling waves with wave speed $c$. Due to the nonlocal diffusion effect, it is more hard to obtain the uniform boundness of solutions. To overcome the difficulties, we construct an invariant cone in a large bounded domain with initial functions being defined on, then pass to the unbounded domain by limiting argument.

This paper is organized as follows. In the following section, we introduce some preliminaries which will be used in the proof of our main results. In Section 3, we will use Schauder's fixed point theorem under the assumption of the compactly supported for the kernel function $J$ and to prove the existence of the traveling waves. Finally, we obtain the nonexistence of the traveling waves by the comparison principle.

\section{Some Preliminaries}

In this section, we will give some useful results for the proof of the existence of the traveling wave solution for the system (4). The traveling wave solution means a solution of the form $(u(x+c t), v(x+c t))$. Let $\xi=x+c t$, then $(u(\xi), v(\xi))$ satisfies

$$
\left\{\begin{array}{l}
c u^{\prime}(\xi)=d_{1} \int_{\mathbb{R}} J(y)(u(\xi-y)-u(\xi)) d y+u(\xi)(1-u(\xi))-\frac{\beta u(\xi) v(\xi)}{u(\xi)+a v(\xi)} \\
c v^{\prime}(\xi)=d_{2} \int_{\mathbb{R}} J(y)(v(\xi-y)-v(\xi)) d y+r v(\xi)\left(1-\frac{v(\xi)}{u(\xi)}\right)
\end{array}\right.
$$


Then we can get characteristic equations

$$
\Delta_{d_{i}, b}(\lambda, c):=d_{i} \int_{\mathbb{R}} J(y) e^{-\lambda y} d y-d_{i}-c \lambda+b,
$$

Where $d_{i}$ and $b$ are non-negative constants. By a direct calculation, for $c>0$ and $\lambda>0$, we can obtain

$$
\begin{gathered}
\Delta_{d_{i}, b}(0, c)=b, \text { especially } \Delta_{d_{i}, 0}(0, c)=0 \text { and } \Delta_{d_{i}, r}(0, c)=r>0, \\
\frac{\partial \Delta_{d_{i}, b}(\lambda, c)}{\partial c}=-\lambda<0, \frac{\partial \Delta_{d_{i}, b}(0, c)}{\partial \lambda}=-c<0 \text { and } \frac{\partial \Delta_{d_{i}, b}(\lambda, c)}{\partial \lambda^{2}}>0 .
\end{gathered}
$$

In view of the above properties of the function $\Delta_{d_{i}, b}(\lambda, c)$, we can get the following lemma.

Lemma 2.1. For any given $0<c<c^{*}$, then $\Delta_{d_{i}, r}(\lambda, c)>0$ for any $\lambda>0$. Moreover, for any $c>c^{*}$, there exist positive constants $\lambda_{2}(c)<\lambda^{*}<\lambda_{1}(c)<\lambda_{3}(c)$ such that

$$
\begin{gathered}
\Delta_{d_{i}, r}(\lambda, c)= \begin{cases}=0, & \lambda=\lambda_{2}(c), \lambda=\lambda_{3}(c), \\
>0, & \lambda \in\left(0, \lambda_{2}(c)\right) \cup\left(\lambda_{3}(c),+\infty\right), \\
<0, & \lambda \in\left(\lambda_{2}(c), \lambda_{3}(c)\right),\end{cases} \\
\Delta_{d_{i}, 0}(\lambda, c)= \begin{cases}=0, & \lambda=\lambda_{1}(c), \\
>0, & \lambda \in\left(\lambda_{1}(c),+\infty\right), \\
<0, & \lambda \in\left(0, \lambda_{1}(c)\right) .\end{cases}
\end{gathered}
$$

In the sequel, we always assume that $c>c^{*}$ and simply denote $\lambda_{i}(c)$ by $\lambda_{i}$ for $i=1,2,3$, respectively.

Definition 2.1. If the functions $u^{+}, v^{+}, u^{-}, v^{-}$satisfy the following inequalities

$$
\begin{gathered}
E\left(u^{+}, v^{+}\right)(\xi):=c\left(u^{+}(\xi)\right)^{\prime}-d_{1}\left(J * u^{+}(\xi)-u^{+}(\xi)\right)-u^{+}(\xi)\left(1-u^{+}(\xi)\right)+\frac{\beta u^{+}(\xi) v^{+}(\xi)}{u^{+}(\xi)+a v^{+}(\xi)} \geq 0, \\
E\left(u^{-}, v^{+}\right)(\xi):=c\left(u^{-}(\xi)\right)^{\prime}-d_{1}\left(J * u^{-}(\xi)-u^{-}(\xi)\right)-u^{-}(\xi)\left(1-u^{-}(\xi)\right)+\frac{\beta u^{-}(\xi) v^{+}(\xi)}{u^{-}(\xi)+a v^{+}(\xi)} \leq 0, \\
E\left(v^{+}, u^{+}\right)(\xi):=c\left(v^{+}(\xi)\right)^{\prime}-d_{2}\left(J * v^{+}(\xi)-v^{+}(\xi)\right)-r v^{+}(\xi)+r \frac{\left(v^{+}(\xi)\right)^{2}}{u^{+}(\xi)} \geq 0 \\
E\left(v^{-}, u^{-}\right)(\xi):=c\left(v^{-}(\xi)\right)^{\prime}-d_{2}\left(J * v^{-}(\xi)-v^{-}(\xi)\right)-r v^{-}(\xi)+r \frac{\left(v^{-}(\xi)\right)^{2}}{u^{-}(\xi)} \leq 0,
\end{gathered}
$$

for $\xi \in \mathbb{R} \backslash D$ with some finite set $D=\left\{\xi_{1}, \xi_{2}, \cdots \cdots \xi_{n}\right\}$ and have no derivatives at $\xi_{i}(i=1,2, \cdots \cdots, n)$, then the functions $\left(u^{+}, v^{+}\right)$and $\left(u^{-}, v^{-}\right)$are called a pair of upper and lower solutions of the system (5).

\section{The Existence of Traveling Waves}

\subsection{Upper and Lower Solutions of The System (5)}

Define

$$
\begin{array}{r}
u^{+}(\xi)= \begin{cases}1, & \xi \leq \frac{2}{\alpha} \ln \frac{\beta}{a}, \\
1-\frac{\beta \delta}{1+a \delta}, & \xi>\frac{2}{\alpha} \ln \frac{\beta}{a},\end{cases} \\
u^{-}(\xi)= \begin{cases}1-\frac{a}{\beta} e^{\alpha \xi}, & \xi \leq \frac{2}{\alpha} \ln \frac{\beta}{a}, \\
1-\frac{\beta}{a}, & \xi>\frac{2}{\alpha} \ln \frac{\beta}{a},\end{cases} \\
v^{+}(\xi)=\min \left\{\frac{a}{\beta^{2}} e^{\lambda_{2} \xi}, 1\right\}, \quad v^{-}(\xi)=\max \left\{0, \frac{a}{\beta^{2}} e^{\lambda_{2} \xi}\left(1-M e^{\eta \xi}\right)\right\},
\end{array}
$$

where

$$
\begin{gathered}
0<\beta<a, \quad 0<\alpha<\lambda_{2} \text { and } d_{1} \int_{\mathbb{R}} J(y) e^{-\alpha y} d y-\alpha c-d_{1}<0 \\
\delta \in\left(0, v^{+}(\xi)\right), \eta \in\left(0, \min \left\{\lambda_{2}, \lambda_{3}-\lambda_{2}\right\}\right)
\end{gathered}
$$

and $M>1$ satisfies 


$$
M=\frac{r a^{2}}{-\beta^{2}(a-\beta) \Delta_{d_{i}, r}\left(\lambda_{2}+\eta, c\right)}+\frac{\beta^{2}}{a}+1
$$

Lemma 3.1. Let $c>c^{*}$, the functions $\left(u^{+}, v^{+}\right)$and $\left(u^{-}, v^{-}\right)$are a pair of upper and lower solutions of the system (5) which are defined as in (11)-(12).

Proof. Firstly, we show that (7) holds. If $\xi \leq \frac{2}{\alpha} \ln \frac{\beta}{a}, u^{+}(\xi)=1$, we can obtain

$$
E\left(u^{+}, v^{+}\right)(\xi)=\frac{\beta v^{+}(\xi)}{1+a v^{+}(\xi)}>0, \text { where } v^{+}(\xi)>0
$$

If $\xi>\frac{2}{\alpha} \ln \frac{\beta}{a}, u^{+}(\xi)=1-\frac{\beta \delta}{1+a \delta}$

$$
\begin{aligned}
E\left(u^{+}, v^{+}\right)(\xi) & =u^{+}(\xi)\left[u^{+}(\xi)-1+\frac{\beta v^{+}(\xi)}{u^{+}(\xi)+a v^{+}(\xi)}\right] \\
& \geq u^{+}\left(\frac{\beta v^{+}(\xi)}{1+a v^{+}(\xi)}-\frac{\beta \delta}{1+a \delta}\right)>0,
\end{aligned}
$$

where we use $\delta<v^{+}(\xi)$ and the function $\frac{\beta x}{1+a x}$ is increasing in $x$, when $\beta>0$.

Next, if $\xi>\frac{2}{\alpha} \ln \frac{\beta}{a}, u^{-}(\xi)=1-\frac{\beta}{a}$, then

$$
\begin{aligned}
E\left(u^{-}, v^{+}\right)(\xi) & =-d_{1}\left(1-\frac{\beta}{a}\right) \int_{\mathbb{R}} J(y) d y+d_{1}\left(1-\frac{\beta}{a}\right)-\left(1-\frac{\beta}{a}\right) \frac{\beta}{a}+\frac{\beta\left(1-\frac{\beta}{a}\right) v^{+}(\xi)}{1-\frac{\beta}{a}+a v^{+}(\xi)} \\
& =\left(1-\frac{\beta}{a}\right)\left[\frac{\beta v^{+}(\xi)}{1-\frac{\beta}{a}+a v^{+}(\xi)}-\frac{\beta}{a}\right] \leq\left(1-\frac{\beta}{a}\right)\left(\frac{\beta}{a}-\frac{\beta}{a}\right)=0 .
\end{aligned}
$$

For $\xi \leq \frac{2}{\alpha} \ln \frac{\beta}{a}<0, u^{-}(\xi)=1-\frac{a}{\beta} e^{\alpha \xi}$ and $v^{+}(\xi)=\min \left\{\frac{a}{\beta^{2}} e^{\lambda_{2} \xi}, 1\right\} \leq \frac{a}{\beta^{2}} e^{\lambda_{2} \xi} \leq \frac{a}{\beta^{2}} e^{\alpha \xi}$ since $0<\alpha<\lambda_{2}$. By easy calculation and the similar above argument, we can get that

$$
\begin{gathered}
1-u^{-}(\xi)-\frac{\beta v^{+}(\xi)}{u^{-}(\xi)+a v^{+}(\xi)}=\frac{a}{\beta} e^{\alpha \xi}-\frac{\beta v^{+}(\xi)}{1-\frac{a}{\beta} e^{\alpha \xi}+a v^{+}(\xi)} \\
\geq \frac{a}{\beta} e^{\alpha \xi}-\frac{\frac{a}{\beta} e^{\alpha \xi}}{1-\frac{a}{\beta} e^{\alpha \xi}+\frac{a^{2}}{\beta^{2}} \alpha^{\alpha \xi}} \geq \frac{a}{\beta} e^{\alpha \xi}-\frac{\frac{a}{\beta} e^{\alpha \xi}}{1-\frac{a}{\beta} e^{\alpha \xi}+\frac{a}{\beta} e^{\alpha \xi}}=0 .
\end{gathered}
$$

Hence, with (13), we obtain

$$
\begin{aligned}
E\left(u^{-}, v^{+}\right)(\xi)= & -c \alpha \frac{a}{\beta} e^{\alpha \xi}-d_{1} \int_{\mathbb{R}} J(y)\left(1-\frac{a}{\beta} e^{\alpha(\xi-y)}\right) d y+d_{1}\left(1-\frac{a}{\beta} e^{\alpha \xi}\right) \\
& -u^{-}(\xi)\left[1-u^{-}(\xi)-\frac{\beta v^{+}(\xi)}{u^{-}(\xi)+a v^{+}(\xi)}\right] \\
= & \frac{a}{\beta} e^{\alpha \xi}\left[d_{1} \int_{\mathbb{R}} J(y) e^{-\alpha y} d y-c \alpha-d_{1}\right]-u^{-}(\xi)\left[1-u^{-}(\xi)-\frac{\beta v^{+}(\xi)}{u^{-}(\xi)+a v^{+}(\xi)}\right] \\
\leq & -u^{-}(\xi)\left[1-u^{-}(\xi)-\frac{\beta v^{+}(\xi)}{u^{-}(\xi)+a v^{+}(\xi)}\right] \leq 0 .
\end{aligned}
$$

So the (8) holds.

Then, we show that (9) holds. It is clear that

$$
v^{+}(\xi)= \begin{cases}1, & \xi \geq \frac{1}{\lambda_{2}} \ln \frac{\beta^{2}}{a}, \\ \frac{a}{\beta^{2}} e^{\lambda_{2} \xi}, & \xi<\frac{1}{\lambda_{2}} \ln \frac{\beta^{2}}{a} .\end{cases}
$$

For $\xi \geq \frac{1}{\lambda_{2}} \ln \frac{\beta^{2}}{a}, v^{+}(\xi)=1$. That is

$$
E\left(v^{+}, u^{+}\right)(\xi)=-r+\frac{r}{u^{+}(\xi)} \geq 0
$$

For $\xi<\frac{1}{\lambda_{2}} \ln \frac{\beta^{2}}{a}, v^{+}(\xi)=\frac{a}{\beta^{2}} e^{\lambda_{2} \xi}$. We can deduce

$$
E\left(v^{+}, u^{+}\right)(\xi) \geq-\frac{a}{\beta^{2}} e^{\lambda_{2} \xi}\left(d_{2} \int_{\mathbb{R}} J(y) e^{-\lambda_{2} y} d y-d_{2}-c \lambda_{2}+r\right)+r \frac{a^{2}}{\beta^{4}} e^{2 \lambda_{2} \xi}
$$


By the definition of $\Delta_{d_{i}, r}(\lambda, c)$ and Lemma 2.1, we can get

$$
E\left(v^{+}, u^{+}\right)(\xi) \geq r \frac{a^{2}}{\beta^{4}} e^{2 \lambda_{2} \xi}>0 .
$$

Lastly, we prove that (10) holds. For $\xi \geq \frac{1}{\eta} \ln \frac{1}{M}, v^{-}(\xi)=0$ and with (14), we get

$$
E\left(u^{-}, v^{-}\right)(\xi):=0
$$

For $\xi<\frac{1}{\eta} \ln \frac{1}{M}<0, v^{-}(\xi)=\frac{a}{\beta^{2}} e^{\lambda_{2} \xi}\left(1-M e^{\eta \xi}\right)$, we have

$$
\begin{aligned}
& E\left(v^{-}, u^{-}\right)(\xi) \\
\leq & c\left[\lambda_{2} \frac{a}{\beta^{2}} e^{\lambda_{2} \xi}-\left(\lambda_{2}+\eta\right) \frac{a}{\beta^{2}} M e^{\left(\lambda_{2}+\eta\right) \xi}\right] \\
& -d_{2}\left[\int_{\mathbb{R}} J(y)\left(\frac{a}{\beta^{2}} e^{\lambda_{2}(\xi-y)}-\frac{a}{\beta^{2}} M e^{\left(\lambda_{2}+\eta\right)(\xi-y)}\right) d y-\frac{a}{\beta^{2}} e^{\lambda_{2} \xi}+\frac{a}{\beta^{2}} M e^{\left(\lambda_{2}+\eta\right) \xi}\right] \\
& -r\left(\frac{a}{\beta^{2}} e^{\lambda_{2} \xi}-\frac{a}{\beta^{2}} M e^{\left(\lambda_{2}+\eta\right) \xi}\right)+r \frac{\left(\frac{a}{\beta^{2}} \lambda_{2} \xi-\frac{a}{\beta^{2}} M e^{\left(\lambda_{2}+\eta\right) \xi}\right)^{2}}{1-\frac{\beta}{a}} \\
\leq & -\frac{a}{\beta^{2}} e^{\lambda_{2} \xi}\left[d_{2} \int_{\mathbb{R}} J(y) e^{-\lambda_{2} y} d y-d_{2}-c \lambda_{2}+r\right]+r \frac{\left(\frac{a}{\beta^{2}} e^{\lambda_{2} \xi}-\frac{a}{\beta^{2}} M e^{\left(\lambda_{2}+\eta\right) \xi}\right)}{1-\frac{\beta}{a}} \frac{a}{\beta^{2}} e^{\lambda_{2} \xi} \\
& +\frac{a}{\beta^{2}} M e^{\left(\lambda_{2}+\eta\right) \xi}\left[d_{2} \int_{\mathbb{R}} J(y) e^{-\left(\lambda_{2}+\eta\right) y} d y-d_{2}-c\left(\lambda_{2}+\eta\right)+r\right] \\
= & \frac{a}{\beta^{2}} M e^{\left(\lambda_{2}+\eta\right) \xi}\left[d_{2} \int_{\mathbb{R}} J(y) e^{-\left(\lambda_{2}+\eta\right) y} d y-d_{2}-c\left(\lambda_{2}+\eta\right)+r\right]+r \frac{a^{3}}{\beta^{4}(a-\beta)} e^{2 \lambda_{2} \xi} \\
& -r \frac{a^{3}}{\beta^{4}(a-\beta)} M e^{\left(2 \lambda_{2}+\eta\right) \xi} \\
\leq & \frac{a}{\beta^{2}} M e^{\left(\lambda_{2}+\eta\right) \xi}\left[d_{2} \int_{\mathbb{R}} J(y) e^{-\left(\lambda_{2}+\eta\right) y} d y-d_{2}-c\left(\lambda_{2}+\eta\right)+r\right]+r \frac{a^{3}}{\beta^{4}(a-\beta)} e^{2 \lambda_{2} \xi} \\
\leq & \frac{a}{\beta^{2}} M e^{\left(\lambda_{2}+\eta\right) \xi}\left[d_{2} \int_{\mathbb{R}} J(y) e^{-\left(\lambda_{2}+\eta\right) y} d y-d_{2}-c\left(\lambda_{2}+\eta\right)+r\right]+r \frac{a^{3}}{\beta^{4}(a-\beta)} \leq 0,
\end{aligned}
$$

where use the facts that $v^{-}(\xi)^{2} \leq v^{-}(\xi) \frac{a}{\beta^{2}} e^{\lambda_{2} \xi},(6)$ and Lemma 2.1, (14)-(15), this completes the proof.

Now we define $A>\max \left\{\frac{1}{\eta} \ln M, \frac{2}{\alpha} \ln \frac{a}{\beta}\right\}$ and a function set

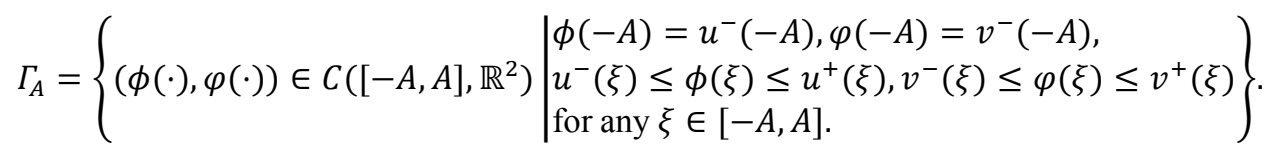

For any $(\phi(\cdot), \varphi(\cdot)) \in C\left([-A, A], \mathbb{R}^{2}\right)$, we define

$$
\hat{\phi}(\xi)=\left\{\begin{array}{ll}
\phi(A), & \xi>A, \\
\phi(\xi), & |\xi| \leq A, \\
u^{-}(-A), & \xi<-A,
\end{array} \quad \hat{\varphi}(\xi)= \begin{cases}\varphi(A), & \xi>A, \\
\varphi(\xi), & |\xi| \leq A, \\
v^{-}(-A), & \xi<-A,\end{cases}\right.
$$

and consider the following initial value problems

$$
\begin{gathered}
c u^{\prime}(\xi)=d_{1} \int_{\mathbb{R}} J(y)(\hat{\phi}(\xi-y)-u(\xi)) d y+\phi(\xi)(1-u(\xi))-\frac{\beta u(\xi) \varphi(\xi)}{\phi(\xi)+a \varphi(\xi)}, \\
c v^{\prime}(\xi)=d_{2} \int_{\mathbb{R}} J(y)(\hat{\varphi}(\xi-y)-v(\xi)) d y+r \varphi(\xi)\left(1-\frac{v(\xi)}{\phi(\xi)}\right),
\end{gathered}
$$

with

$$
u(-A)=u^{-}(-A), \quad v(-A)=v^{-}(-A) .
$$

Obviously, the problems (16)-(18) admit a unique solution $\left(u_{A}(\cdot), v_{A}(\cdot)\right)$ satisfying $u_{A}(\cdot) \in C^{1}([-A, A])$ and $v_{A}(\cdot) \in$ $C^{1}([-A, A])$. Then, we define an operator $\mathcal{F}=\left(\mathcal{F}_{1}, \mathcal{F}_{2}\right): \Gamma_{A} \rightarrow C([-A, A])$ by $\mathcal{F}_{1}[\phi, \varphi](\xi)=u_{A}(\xi)$ and $\mathcal{F}_{2}[\phi, \varphi](\xi)=v_{A}(\xi)$ for $\xi \in[-A, A]$.

Lemma 3.2. The operator $\mathcal{F}$ maps $\Gamma_{A}$ into $\Gamma_{A}$.

Proof. For any $(\phi(\cdot), \varphi(\cdot)) \in \Gamma_{A}$, we should prove that

$$
\mathcal{F}_{1}[\phi, \varphi](-A)=u^{-}(-A), \mathcal{F}_{2}[\phi, \varphi](-A)=v^{-}(-A),
$$


and

$$
u^{-}(\xi) \leq \mathcal{F}_{1}[\phi, \varphi](\xi) \leq u^{+}(\xi), v^{-}(\xi) \leq \mathcal{F}_{2}[\phi, \varphi](\xi) \leq v^{+}(\xi) \quad \text { for any } \quad \xi \in[-A, A] .
$$

By the definition of the operator $\mathcal{F}$, it is obvious to see that

$$
\mathcal{F}_{1}[\phi, \varphi](-A)=u_{A}(-A)=u^{-}(-A), \mathcal{F}_{2}[\phi, \varphi](-A)=v_{A}(-A)=v^{-}(-A) .
$$

Then according to Lemma 3.1 and direct calculation, we can directly obtain

$$
u^{-}(\xi) \leq \mathcal{F}_{1}[\phi, \varphi](\xi) \leq u^{+}(\xi), v^{-}(\xi) \leq \mathcal{F}_{2}[\phi, \varphi](\xi) \leq v^{+}(\xi) \quad \text { for any } \quad \xi \in[-A, A] .
$$

This ends the proof.

Lemma 3.3. The operator $\mathcal{F}: \Gamma_{A} \rightarrow \Gamma_{A}$ is completely continuous.

Proof. We first show that $\mathcal{F}$ is continuous. By a direct calculation, we get that

$$
\begin{aligned}
\mathcal{F}_{1}[\phi, \varphi]=u_{A}(\xi)= & u^{-}(-A) \exp \left\{-\frac{1}{c} \int_{-A}^{\xi}\left[d_{1}+\phi(s)+\frac{\beta \varphi(s)}{\phi(s)+a \varphi(s)}\right] d s\right\} \\
& +\frac{1}{c} \int_{-A}^{\xi} \exp \left\{-\frac{1}{c} \int_{\eta}^{\xi}\left[d_{1}+\phi(s)+\frac{\beta \varphi(s)}{\phi(s)+a \varphi(s)}\right] d s\right\}\left[f_{\phi}(\eta)+\phi(\eta)\right] d \eta,
\end{aligned}
$$

and

$$
\begin{aligned}
\mathcal{F}_{2}[\phi, \varphi]=v_{A}(\xi)= & v^{-}(-A) \exp \left\{-\frac{1}{c} \int_{-A}^{\xi}\left[d_{2}+r \frac{\varphi(s)}{\phi(s)}\right] d s\right\} \\
& +\frac{1}{c} \int_{-A}^{\xi} \exp \left\{-\frac{1}{c} \int_{\eta}^{\xi}\left[d_{2}+r \frac{\varphi(s)}{\phi(s)}\right] d s\right\}\left[d g_{\varphi}(\eta)+r \varphi(\eta)\right] d \eta,
\end{aligned}
$$

where

$$
f_{\phi}(\eta)=\int_{-\infty}^{-A} J(\eta-y) u^{-}(y) d y+\int_{-A}^{A} J(\eta-y) \phi(y) d y+\int_{A}^{+\infty} J(\eta-y) \phi(A) d y,
$$

and

$$
g_{\varphi}(\eta)=\int_{-\infty}^{-A} J(\eta-y) v^{-}(y) d y+\int_{-A}^{A} J(\eta-y) \varphi(y) d y+\int_{A}^{+\infty} J(\eta-y) \varphi(A) d y .
$$

For $\forall\left(\phi_{1}(\cdot), \varphi_{1}(\cdot)\right),\left(\phi_{2}(\cdot), \varphi_{2}(\cdot)\right) \in \Gamma_{A}$, we have that

$$
\begin{aligned}
\left|f_{\phi_{1}}(\eta)-f_{\phi_{2}}(\eta)\right| & \leq\left|\int_{-A}^{A} J(\eta-y)\left[\phi_{1}(y)-\phi_{2}(y)\right] d y\right|+\left|\int_{A}^{+\infty} J(\eta-y)\left[\phi_{1}(A)-\phi_{2}(A)\right] d y\right| \\
& \leq 2 \max _{y \in[-A, A]}\left|\phi_{1}(y)-\phi_{2}(y)\right|,
\end{aligned}
$$

and

$$
\left|g_{\varphi_{1}}(\eta)-g_{\varphi_{2}}(\eta)\right| \leq 2 \max _{y \in[-A, A]}\left|\varphi_{1}(y)-\varphi_{2}(y)\right| .
$$

Combining with the continuity of the compound function, $\mathcal{F}$ is continuous.

Next we confirm that $\mathcal{F}$ is compacted, thus we should prove that for any bounded subset $\Omega \subset \Gamma_{A}, \mathcal{F}(\Omega)$ is precompact. By the definition of $\mathcal{F}$, we have that for all $\left(u_{A}, v_{A}\right) \in \mathcal{F}(\Omega)$, there exists $(\phi, \varphi) \in \Omega$ such that

$$
\mathcal{F}[\phi, \varphi](\xi)=\left(u_{A}, v_{A}\right)(\xi), \forall \xi \in[-A, A] .
$$

Since $(\phi, \varphi) \in \Omega$, in (19) and (20), we obtain that

$$
\left|u_{A}(\xi)\right| \leq M_{1} \text { and }\left|v_{A}(\xi)\right| \leq M_{1}, \forall \xi \in[-A, A]
$$

where $M_{1}>0$ is a constant. That is, $\mathcal{F}(\Omega)$ is uniformly bounded. Further, according to the equations (16), (17) and the above inequality, then there exists some constant $M_{2}>0$ such that

$$
\left|u_{A}^{\prime}(\xi)\right| \leq M_{2} \text { and }\left|v_{A}^{\prime}(\xi)\right| \leq M_{2}, \forall \xi \in[-A, A] .
$$

So we can get that $\mathcal{F}(\Omega)$ is equicontinuous. By Arzela-Ascoli Theorem, then we have that $\mathcal{F}(\Omega)$ is precompact. Thus we establish that $\mathcal{F}: \Gamma_{A} \rightarrow \Gamma_{A}$ is completely continuous with respect to the maximum norm.

Theorem 3.1. The operator $\mathcal{F}$ has a fixed point in $\Gamma_{A}$.

Proof. By the definition of $\Gamma_{A}$, it is easy to see that $\Gamma_{A}$ is closed and convex. Thus, according to Lemma 3.3 and using Schauder's fixed point theorem, there exists $\left(u_{A}^{*}(\cdot), v_{A}^{*}(\cdot)\right) \in \Gamma_{A}$ such that 


$$
\left(u_{A}^{*}(\xi), v_{A}^{*}(\xi)\right)=\mathcal{F}\left[u_{A}^{*}, v_{A}^{*}\right](\xi), \forall \xi \in[-A, A] .
$$

To obtain the existence of solutions for (5), we need some estimates about $\left(u_{A}^{*}(\cdot), v_{A}^{*}(\cdot)\right)$. For the sake of convenience, we use $\left(u_{A}(\cdot), v_{A}(\cdot)\right)$ instead of $\left(u_{A}^{*}(\cdot), v_{A}^{*}(\cdot)\right)$.

Assumption 3.1. $\left(K_{3}\right)$ The kernel function $J$ is compactly supported.

Lemma 3.4. Assume that $\left(K_{1}\right)-\left(K_{3}\right)$ hold, then there exists some constant $C>0$ such that

$$
\left\|u_{A}\right\|_{C^{1,1}([-B, B])}<C \text { and }\left\|v_{A}\right\|_{C^{1,1}([-B, B])}<C
$$

for any $0<B<A$, where $A>\max \left\{\frac{1}{\eta} \ln M, \frac{2}{\alpha} \ln \frac{a}{\beta}\right\}$.

Proof. By Theorem 3.1, we have that $\left(u_{A}(\cdot), v_{A}(\cdot)\right)$ satisfies

$$
c u_{A}^{\prime}(\xi)=d_{1}\left(\int_{\mathbb{R}} J(y) \hat{u}_{A}(\xi-y) d y-u_{A}(\xi)\right)+u_{A}(\xi)\left[1-u_{A}(\xi)\right]-\frac{\beta u_{A}(\xi) v_{A}(\xi)}{u_{A}(\xi)+a v_{A}(\xi)^{\prime}}
$$

and

$$
c v^{\prime}{ }_{A}(\xi)=d_{2}\left(\int_{\mathbb{R}} J(y) \hat{v}_{A}(\xi-y) d y-v_{A}(\xi)\right)+r v_{A}(\xi)\left(1-\frac{v_{A}(\xi)}{u_{A}(\xi)}\right)
$$

where

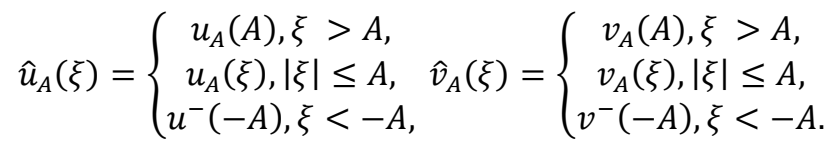

Following that $1-\frac{\beta}{a} \leq u_{A}(\xi) \leq 1,0<v_{A}(\xi) \leq 1$ for $\xi \in[-B, B]$, we have

$$
\begin{aligned}
\left|u_{A}^{\prime}(\xi)\right| & \leq \frac{d_{1}}{c}\left|\int_{\mathbb{R}} J(y) \hat{u}_{A}(\xi-y) d y\right|+\frac{d_{1}}{c}\left|u_{A}(\xi)\right|+\frac{1}{c}\left|u_{A}(\xi)\left(1-u_{A}(\xi)\right)\right|+\frac{\beta}{c} \frac{\left|u_{A}(\xi) v_{A}(\xi)\right|}{\left|u_{A}(\xi)+a v_{A}(\xi)\right|} \\
& \leq \frac{2 d_{1}+\frac{1}{4}+\frac{a \beta}{a-\beta}}{c}
\end{aligned}
$$

and

$$
\begin{aligned}
\left|v_{A}^{\prime}(\xi)\right| & \leq \frac{d_{2}}{c}\left|\int_{\mathbb{R}} J(y) \hat{v}_{A}(\xi-y) d y\right|+\frac{d_{2}}{c}\left|v_{A}(\xi)\right|+\frac{r}{c}\left|v_{A}(\xi)\left(1-\frac{v_{A}(\xi)}{u_{A}(\xi)}\right)\right| \\
& \leq \frac{2 d_{2}+r / 4}{c} .
\end{aligned}
$$

So there exists some constant $C_{1}>0$ such that $\left\|u_{A}\right\|_{C^{1}([-B, B])}<C_{1}$ and $\left\|v_{A}\right\|_{C^{1}([-B, B])}<C_{1}$. It is obvious to obtain that

$$
\left|u_{A}(\xi)-u_{A}(\eta)\right|<C_{1}|\xi-\eta| \text { and }\left|v_{A}(\xi)-v_{A}(\eta)\right|<C_{1}|\xi-\eta|
$$

for any $\xi, \eta \in[-B, B]$. In view of (3.11), we have

$$
\begin{aligned}
c\left|u_{A}^{\prime}(\xi)-u_{A}^{\prime}(\eta)\right| \leq & d_{1}\left|\int_{\mathbb{R}} J(y)\left[\hat{u}_{A}(\xi-y)-\hat{u}_{A}(\eta-y)\right] d y\right|+d_{1}\left|u_{A}(\xi)-u_{A}(\eta)\right| \\
& +\left|u_{A}(\xi)\left[1-u_{A}(\xi)\right]-u_{A}(\eta)\left[1-u_{A}(\eta)\right]\right| \\
& +\frac{\beta a}{(a-\beta)}\left|u_{A}(\xi) v_{A}(\xi)-u_{A}(\eta) v_{A}(\eta)\right| \\
:= & d_{1} U_{1}+d_{1} U_{2}+U_{3}+\frac{\beta a}{(a-\beta)} U_{4} .
\end{aligned}
$$

By the conditions $\left(K_{1}\right)-\left(K_{3}\right)$, we can assume that $L$ is its Lipschitz constant and $R$ is the radius of supp $J$. Then, we have

$$
\begin{aligned}
U_{1}= & \left|\int_{-R}^{+R} J(y) \hat{u}_{A}(\xi-y) d y-\int_{-R}^{+R} J(y) \hat{u}_{A}(\eta-y) d y\right| \\
= & \left|\int_{\xi-R}^{\xi+R} J(\xi-y) \hat{u}_{A}(y) d y-\int_{\eta-R}^{\eta+R} J(\eta-y) \hat{u}_{A}(y) d y\right| \\
\leq & \left|\int_{\eta+R}^{\xi+R} J(\xi-y) \hat{u}_{A}(y) d y\right|+\left|\int_{\xi-R}^{\eta-R} J(\xi-y) \hat{u}_{A}(y) d y\right| \\
& +\left|\int_{\eta-R}^{\eta+R}[J(\xi-y)-J(\eta-y)] \hat{u}_{A}(y) d y\right| \\
\leq & 2\left(\|J\|_{L^{\infty}}+R L\right)|\xi-\eta|,
\end{aligned}
$$




$$
\begin{aligned}
U_{3} & =\left|u_{A}(\xi)-u_{A}^{2}(\xi)-u_{A}(\eta)+u_{A}^{2}(\eta)\right| \\
& \leq\left|u_{A}(\xi)-u_{A}(\eta)\right|+\left|u_{A}^{2}(\xi)-u_{A}^{2}(\eta)\right| \\
& \leq 3\left|u_{A}(\xi)-u_{A}(\eta)\right|,
\end{aligned}
$$

and

$$
\begin{aligned}
U_{4} & =\left|u_{A}(\xi) v_{A}(\xi)-u_{A}(\eta) v_{A}(\eta)\right| \\
& \leq\left|u_{A}(\xi)-u_{A}(\eta)\right|\left|v_{A}(\xi)\right|+\left|u_{A}(\eta)\right|\left|v_{A}(\xi)-v_{A}(\eta)\right| \\
& \leq\left|u_{A}(\xi)-u_{A}(\eta)\right|+\left|v_{A}(\xi)-v_{A}(\eta)\right| .
\end{aligned}
$$

Combining (23) and (24), we obtain that there exists some constant $L_{2}>0$ such that

$$
\left|u_{A}^{\prime}(\xi)-u_{A}^{\prime}(\eta)\right| \leq L_{2}|\xi-\eta| \text {. }
$$

Then applying with (19), we also have

$$
\begin{aligned}
c\left|v_{A}^{\prime}(\xi)-v_{A}^{\prime}(\eta)\right| \leq & d_{2}\left|\int_{\mathbb{R}} J(y)\left[\hat{v}_{A}(\xi-y)-\hat{v}_{A}(\eta-y)\right] d y\right|+d\left|v_{A}(\xi)-v_{A}(\eta)\right| \\
& +r\left|v_{A}(\xi)\left(1-\frac{v_{A}(\xi)}{u_{A}(\xi)}\right)-v_{A}(\eta)\left(1-\frac{v_{A}(\eta)}{u_{A}(\eta)}\right)\right| \\
:= & d_{2} V_{1}+d_{2} V_{2}+r V_{3} .
\end{aligned}
$$

Similar to the same argument of $U_{1}$, we have that

$$
\begin{aligned}
V_{1} & =\left|\int_{\mathbb{R}} J(y) \hat{v}_{A}(\xi-y) d y-\int_{\mathbb{R}} J(y) \hat{v}_{A}(\eta-y) d y\right| \\
& \leq 2\left(\|J\|_{L^{\infty}}+R L\right)|\xi-\eta| .
\end{aligned}
$$

Then, by (23), (25) with

$$
\begin{aligned}
V_{3} & \leq\left|v_{A}(\xi)-v_{A}(\eta)\right|+\left|\frac{v_{A}^{2}(\xi) u_{A}(\eta)-v_{A}^{2}(\eta) u_{A}(\xi)}{u_{A}(\xi) u_{A}(\eta)}\right| \\
& \leq\left|v_{A}(\xi)-v_{A}(\eta)\right|+\frac{\left|v_{A}^{2}(\xi)-v_{A}^{2}(\eta)\right|}{\left|u_{A}(\xi)\right|}+\frac{\left|v_{A}^{2}(\eta)\right|\left|u_{A}(\eta)-u_{A}(\xi)\right|}{\left|u_{A}(\xi) u_{A}(\eta)\right|} \\
& \leq\left|v_{A}(\xi)-v_{A}(\eta)\right|+\frac{2 a}{a-\beta}\left|v_{A}(\xi)-v_{A}(\eta)\right|+\frac{a^{2}}{(a-\beta)^{2}}\left|u_{A}(\eta)-u_{A}(\xi)\right| \\
& \leq \frac{3 a-\beta}{a-\beta}\left|v_{A}(\xi)-v_{A}(\eta)\right|+\frac{a^{2}}{(a-\beta)^{2}}\left|u_{A}(\eta)-u_{A}(\xi)\right|,
\end{aligned}
$$

we can get that

$$
\left|v_{A}^{\prime}(\xi)-v^{\prime}{ }_{A}(\eta)\right| \leq L_{2}|\xi-\eta|
$$

for any $\xi, \eta \in[-B, B]$. So we have obtained that there exists a constant $C>0$ for any $B$ satisfying $B<A$ independent of $A>\max \left\{\frac{1}{\eta} \ln M, \frac{2}{\alpha} \ln \frac{a}{\beta}\right\}$ such that

$$
\left\|u_{A}\right\|_{C^{1,1}([-B, B])}<C \text { and }\left\|v_{A}\right\|_{C^{1,1}([-B, B])}<C
$$

\subsection{Existence of Traveling Waves}

Theorem 3.2. Assume that $\left(K_{1}\right)-\left(K_{3}\right)$ hold. For any $c>c^{*}$, there exists a pair function $(\tilde{u}(\xi), \tilde{v}(\xi))$ satisfying (5), $(\tilde{u}(-\infty), \tilde{v}(-\infty))=(1,0)$ and $(\tilde{u}(+\infty), \tilde{v}(+\infty))=(k, k)$, where $k=1-\frac{\beta}{a+1}$.

Proof. Choosing an increasing sequence $\left\{A_{n}\right\}_{n=1}^{+\infty}$ such that $\lim _{n \rightarrow+\infty} A_{n}=+\infty$ and $A_{n}>\max \left\{\frac{1}{\eta} \ln M, \frac{2}{\alpha} \ln \frac{a}{\beta}\right\}$ for each $n$. For every $c>c^{*}$, there exists $\left(u_{A_{n}}, v_{A_{n}}\right) \in \Gamma_{A_{n}}$ which satisfies Lemma 3.4 and equations (21), (22) in $\xi \in\left[-A_{n}, A_{n}\right]$. According to the estimates for the sequence $\left\{\left(u_{A_{n}}, v_{A_{n}}\right)\right\}$ in Lemma 3.4, we can extract a subsequence by a standard diagonal extract argument, denoted by $\left\{\left(u_{A_{n_{k}}}, v_{A_{n_{k}}}\right)\right\}_{k \in N}$, tending towards $(\tilde{u}, \tilde{v}) \in C^{1}(\mathbb{R})$ in the following topologies

$$
u_{A_{n_{k}}} \rightarrow \tilde{u} \text { and } v_{A_{n_{k}}} \rightarrow \tilde{v} \text { in } C_{l o c}^{1}(\mathbb{R}) \text { as } k \rightarrow+\infty .
$$

By the assumption of the kernel function $J(y)$ and applying the dominated convergence theorem, we can obtain that

$$
\lim _{k \rightarrow+\infty} \int_{\mathbb{R}} J(y) \hat{u}_{A_{n_{k}}}(\xi-y) d y=\int_{\mathbb{R}} J(y) \tilde{u}(\xi-y) d y,
$$

and 


$$
\lim _{k \rightarrow+\infty} \int_{\mathbb{R}} J(y) \hat{v}_{A_{n_{k}}}(\xi-y) d y=\int_{\mathbb{R}} J(y) \tilde{v}(\xi-y) d y
$$

for any $\xi \in \mathbb{R}$. Then, it is easy to show that $(\tilde{u}, \tilde{v})$ satisfies system (5) and

$$
u^{-}(\xi) \leq \tilde{u}(\xi) \leq u^{+}(\xi), \quad v^{-}(\xi) \leq \tilde{v}(\xi) \leq v^{+}(\xi) .
$$

By the above inequality, we can further get that $(\tilde{u}, \tilde{v})$ satisfies $(\tilde{u}(-\infty), \tilde{v}(-\infty))=(1,0)$, and

$$
1-\frac{\beta}{a} \leq \liminf _{\xi \rightarrow+\infty} \tilde{u}(\xi) \leq \limsup _{\xi \rightarrow+\infty} \tilde{u}(\xi) \leq 1-\frac{\beta \delta}{1+a \delta}, 0 \leq \liminf _{\xi \rightarrow+\infty} \widetilde{v}(\xi) \leq \limsup _{\xi \rightarrow+\infty} \widetilde{v}(\xi) \leq 1
$$

Since $\limsup _{\xi \rightarrow+\infty} \widetilde{u}(\xi) \leq 1-\frac{\beta \delta}{1+a \delta}$ and $\liminf _{\xi \rightarrow+\infty} \tilde{v}(\xi)>0$, there exist $\xi>\xi_{0}$ and $\delta_{0} \in\left(0,1-\frac{\beta}{a}\right)$ such that $\tilde{u}(\xi) \leq 1-\frac{\beta \delta_{0}}{1+a \delta_{0}}$ and $\delta_{0} \leq \tilde{v}(\xi)$ for any $\xi>\xi_{0}$, then by (26), we get that

$$
\delta_{0}<\tilde{u}(\xi) \leq 1-\frac{\beta \delta_{0}}{1+a \delta_{0}} \text { and } \delta_{0} \leq \tilde{v}(\xi) \leq 1
$$

for any $\xi>\xi_{0}$. Now, we introduce a sequence $\left\{\gamma_{n}\right\}_{n \geq 0}$ defined by

$$
\left\{\begin{array}{l}
\gamma_{0}=1, \gamma_{1}=\delta_{0} \\
1-\gamma_{n+1}=\frac{\beta \gamma_{n}}{\gamma_{n-1}+a \gamma_{n}}, n \geq 1 .
\end{array}\right.
$$

By direct calculation, we can get that the sequences $\left\{\gamma_{2 n}\right\}_{n \geq 0}$ and $\left\{\gamma_{2 n+1}\right\}_{n \geq 0}$ are adjacent. They converge to $k$ and satisfy for each $n \geq 0$,

$$
\gamma_{1}<\gamma_{3}<\cdots<\gamma_{2 n+1}<\cdots<1-\frac{\beta}{1+a}<\cdots<\gamma_{2 n}<\cdots<\gamma_{2}<\gamma_{0} .
$$

Next, we prove that

$$
\gamma_{2 n+1} \leq \tilde{u}(\xi) \leq \gamma_{2 n+2} \text { and } \gamma_{2 n+1} \leq \tilde{v}(\xi) \leq \gamma_{2 n}
$$

for all $n \geq 0$ and $\xi>\xi_{0}$. According to the inequality (27), this inequality (28) holds true for $n=0$. Let us now argue by induction on $n$. Assume that (28) hold true for all $n \geq 1$ and let us prove that (28) holds true for $n+1$. Since $\tilde{u}(\xi) \leq \gamma_{2 n+2}$, then $\tilde{v}(\xi)$ satisfies

$$
c \tilde{v}^{\prime}(\xi)-d_{2} \int_{\mathbb{R}} J(y)[\tilde{v}(\xi-y)-\tilde{v}(\xi)] d y-r \tilde{v}(\xi)\left(1-\frac{\tilde{v}(\xi)}{\gamma_{2 n+2}}\right) \leq 0 \text { for } \xi \geq \xi_{0} .
$$

which means that $\tilde{v}(\xi)$ is the subsolution of the equation

$$
c \tilde{v}^{\prime}(\xi)-d_{2} \int_{\mathbb{R}} J(y)[\tilde{v}(\xi-y)-\tilde{v}(\xi)] d y-r \tilde{v}(\xi)\left(1-\frac{\tilde{v}(\xi)}{\gamma_{2 n+2}}\right)=0 \text { for } \xi \geq \xi_{0} .
$$

Since $\gamma_{2 n+2}$ is a solution of the equation (29), we can get that $\tilde{v}(\xi) \leq \gamma_{2 n+2}$ for all $\xi \geq \xi_{0}$. Then we can get that $\tilde{u}(\xi)$ satisfies

$$
c \tilde{u}^{\prime}(\xi)-d_{1} \int_{\mathbb{R}} J(y)[\tilde{u}(\xi-y)-\tilde{u}(\xi)] d y-\tilde{u}(\xi)[1-\tilde{u}(\xi)]+\frac{\beta \gamma_{2 n+2} \tilde{u}(\xi)}{\gamma_{2 n-1}+a \gamma_{2 n+2}} \geq 0 \text { for } \xi \geq \xi_{0} .
$$

which means, $\tilde{u}(\xi)$ is the supersolution of the equation

$$
c \tilde{u}^{\prime}(\xi)-d_{1} \int_{\mathbb{R}} J(y)[\tilde{u}(\xi-y)-\tilde{u}(\xi)] d y-\tilde{u}(\xi)[1-\tilde{u}(\xi)]+\frac{\beta \gamma_{2 n+2} \tilde{u}(\xi)}{\gamma_{2 n-1}+a \gamma_{2 n+2}}=0 \text { for } \xi \geq \xi_{0} .
$$

Using the fact that $1-\gamma_{2 n+3}=\frac{\beta \gamma_{2 n+2}}{\gamma_{2 n+1}+a \gamma_{2 n+2}}$ for $n \geq 0$, we can get that $\tilde{u}(\xi) \geq \gamma_{2 n+3}$ for $\xi \geq \xi_{0}$. By the same arguments as before and $\tilde{u}(\xi) \geq \gamma_{2 n+3}$, one can easy to conclude that $\tilde{v}(\xi) \geq \gamma_{2 n+3}$ for $\xi \geq \xi_{0}$. Then we can use the result to get that $\tilde{u}(\xi) \leq \gamma_{2 n+4}$ for $\xi \geq \xi_{0}$. Thus (28) holds true for $n+1$. Letting $n \rightarrow+\infty$ of (28), we can obtain that

$$
\tilde{u}(\xi) \equiv 1-\frac{\beta}{a+1}, \tilde{v}(\xi) \equiv 1-\frac{\beta}{a+1}
$$

for $\xi \geq \xi_{0}$. So we can get $\tilde{u}(+\infty)=\tilde{v}(+\infty)=1-\frac{\beta}{a+1}$. This completes the proof of the theorem.

\section{Nonexistence of Traveling Waves}

In this section, we will establish the nonexistence of traveling waves for (5) when $0<c<c^{*}$. When the initial value is given, the traveling wave system becomes a single fisher's equation. So we can use the comparison principle to prove the non-existence of traveling wave solutions. Firstly, we consider an associated Cauchy problem for Fisher's KPP equation with the nonlocal diffusion 


$$
\left\{\begin{array}{l}
\frac{\partial U(x, t)}{\partial t}=d(J * U(x, t)-U(x, t))+U(x, t)(1-U(x, t)) \\
U(x, 0)=\chi(x), x \in \mathbb{R}
\end{array}\right.
$$

where $J$ satisfies condition $\left(K_{1}\right)-\left(K_{2}\right), \mathrm{d}$ is a constant, and the initial function $\chi(x)$ is uniformly continuous and bounded. In view of [18], we have the following Lemmas of system (30).

Lemma 4.1. Assume that $0 \leq \chi(x) \leq 1$. Then system (30) admits a solution for all $x \in \mathbb{R}$ and $t>0$. If $W(x, 0)$ is uniformly continuous and bounded, and $W(x, t)$ satisfies

$\left\{\begin{array}{l}\left.\frac{\partial W(x, t)}{\partial t} \geq(\leq) d(J * W(x, t)-W(x, t))+W(x, t)\right)(1-W(x, t)), \\ W(x, 0) \geq(\leq) \chi(x), x \in \mathbb{R},\end{array}\right.$

then there holds

$$
W(x, t) \geq(\leq) U(x, t), x \in \mathbb{R}, t>0 .
$$

Lemma 4.2. Assume that $\chi(x)>0$. Then for any $0<c<$ $c^{*}$, we have

$$
\liminf _{t \rightarrow+\infty} \inf _{|x|<c t} U(x, t)=\limsup _{t \rightarrow+\infty} \sup _{|x|<c t} U(x, t)=1 .
$$

Lemmas 4.1-4.2 can be directly derived from the theorem 2.3 and 3.5 of [18].

Theorem 4.1. For any speed $0<c<c^{*}$, there exists no nontrivial positive solution $(u(\xi), u(\xi))$ of (5) satisfying

$$
\lim _{\xi \rightarrow-\infty}(u(\xi), v(\xi))=(1,0) \text { and } \lim _{\xi \rightarrow+\infty}(u(\xi), v(\xi))=(k, k) .
$$

Proof. By contradiction, we suppose that there exists some $c_{1}<c^{*}$ such that system (5) has a positive solution $(u(\xi), v(\xi))$ satisfying $(31)$. Then $u(\xi)$ is bounded on $\mathbb{R}$. We can find a positive constant $R_{2}$ such that $v(\xi)=v\left(x+c_{1} t\right)$ satisfies

$$
\left\{\begin{array}{l}
\left.\frac{\partial v(x, t)}{\partial t} \geq d_{2}(J * v(x, t)-v(x, t))+r v(x, t)\right)\left(1-R_{2} v(x, t)\right), \\
v(x, 0)=v(x)>0 .
\end{array}\right.
$$

Let $x(t)=-\frac{\left(c_{1}+c^{*}\right)}{2} t$. Then $|x(t)|=\frac{\left(c_{1}+c^{*}\right)}{2}|t|<c^{*} t$. From Lemmas 4.1-4.2, we obtain

$$
\liminf _{t \rightarrow+\infty} \inf _{|x|=\frac{\left(c_{1}+c^{*}\right)}{2} t} v(x, t) \geq \frac{1}{R_{2}}>0
$$

On the other hand, let $x(t)+c_{1} t=\frac{\left(c_{1}-c^{*}\right)}{2} t$, then

$$
\xi=x+c_{1} t \rightarrow-\infty \text { as } t \rightarrow+\infty,
$$

and

$$
\limsup _{t \rightarrow+\infty} v(x(t), t)=\lim _{\xi \rightarrow-\infty} v(\xi)=0,
$$

which is a contradiction. Hence, the proof is completed.

\section{Acknowledgements}

The second author was supported by National Natural Science Foundation of China (11701341) and Natural
Science Foundation of Shandong Province (ZR2017BA005).

\section{References}

[1] P. Bates, P. Fife, X. Ren and X. Wang, Traveling waves in a convolution model for phase transitions, Arch. Ration. Mech. Anal., 1997, 138: 105-136.

[2] J. Carr and A. Chmaj, Uniqueness of travelling waves for nonlocal monostable equations, Proc. Amer. Math. Soc., 2004, 132: $2433-2439$.

[3] S.-S. Chen and J.-P. Shi, Global stability in a diffusive Holling-Tanner predator-prey model, Appl. Math. Lett., 2012, 25: 614-618.

[4] X. Chen, Existence, uniqueness, and asymptotic stability of traveling waves in nonlocal evolution equations, $A d v$. Differential Equations, 1997, 2: 125-160.

[5] H. Cheng and R. Yuan, The spreading property for a preypredator reaction-diffusion system with fractional diffusion, Frac. Calc. Appl. Anal., 2015, 18: 565-579.

[6] H. Cheng and R. Yuan, Existence and stability of traveling waves for Leslie-Gower predator-prey system with nonlocal diffusion. Discrete Contin. Dyn. Syst., 2017, 37 (10): 54335454 .

[7] H. Cheng and R. Yuan, Traveling waves of some HollingTanner predator-prey system with nonlocal diffusion. Appl. Math. Comput., 2018, 338 (1): 12-24.

[8] C. Conley and R. Gardner, An application of the generalized Morse index to travelling wave solutions of a competitive reaction-diffusion model, Indiana Univ. Math. J., 1984, 33: 319-343.

[9] J. Coville and L. Dupaigne, Propagation speed of travelling fronts in non local reaction-diffusion equations, Nonlinear Anal., 2005, 60: 797-819.

[10] J. Coville and L. Dupaigne, On a non-local equation arising in population dynamics, Proc. Roy. Soc. Edinburgh Sect. A, 2007, 137: 727-755.

[11] A. Ducrot, Convergence to generalized transition waves for some Holling-Tanner prey-predator reaction-diffusion system, J. Math. Pures Appl., 2013, 100: 1-15.

[12] S. R. Dunbar, Traveling wave solutions of diffusive LotkaVolterra equations: a heteroclinic connection in $R^{4}$, Trans. Amer. Math. Soc., 1984, 286: 557-594.

[13] W. Feng, W.-H. Ruan and X. Lu, On existence of wavefront solutions in mixed monotone reaction-diffusion systems, Discrete Contin. Dyn. Syst. Ser. B, 2016, 21: 815-836.

[14] R. A. Gardner, Existence and stability of travelling wave solutions of competition models: A degree theoretic approach, J. Differential Equations, 1982, 44: 343-364.

[15] C. S. Holling, The functional response of predators to prey density and its role in mimicry and population regulation, Mem. Entomol. Soci. Can., 1965, 97: 5-60.

[16] Y.-L. Huang and G. Lin, Traveling wave solutions in a diffusive system with two preys and one predator, J. Math. Anal. Appl., 2014, 418: 163-184. 
[17] J. Huang and X. Zou, Traveling wavefronts in diffusive and cooperative Lotka-Volterra system with delays, J. Math. Anal. Appl., 2002, 271: 455-466. Y. Jin, X.-Q.

[18] Y. Jin, X.-Q. Zhao, Spatial dynamics of a periodic population model with dispersal. Nonlinearity, 2009, 22: 1167-1189.

[19] J. Kanel and L. Zhou, Existence of wave front solutions and estimates of wave speed for a competition-diffusion system, Nonlinear Anal., 1996, 27: 579-587.

[20] G. Lin, Invasion traveling wave solutions of a predator-prey system, Nonlinear Anal., 2014, 96: 47-58.

[21] S.-W. Ma, Traveling wavefronts for delayed reaction-diffusion systems via a fixed point theorem, J. Differential Equations, 2001, 171: 294-314.

[22] M. R. Owen and M. A. Lewis, How predation can slow, stop or reverse a prey invasion, Bull. Math. Biol., 2001, 63: 655684 .

[23] S. Pan, Traveling wave fronts of delayed non-local diffusion systems without quasimonotonicity, J. Math. Anal. Appl., 2008, 346: 415-424.

[24] S. Pan, W.-T. Li and G. Lin, Travelling wave fronts in nonlocal delayed reaction-diffusion systems and applications, Z. Angew. Math. Phys., 2009, 60: 377-392.
[25] R. Peng and M.-X. Wang, Positive steady states of the Holling-Tanner prey-predator model with diffusion, Proc. Roy. Soc. Edinburgh Sect. A, 2005, 135: 149-164.

[26] S. Petrovskii, A. Morozov and B.-L. Li, Regimes of biological invasion in a predator-prey system with the Allee effect, Bull. Math. Biol., 2005, 67: 637-661.

[27] T. Su and G-B. Zhang, Invasion traveling waves for a discrete diffusive ratio-dependent predator-prey model, in press.

[28] J. T. Tanner, The stability and the intrinsic growth rates of prey and predator populations, Ecology, 1975, 855-867.

[29] M. M. Tang and P. C. Fife, Propagating fronts for competing species equations with diffusion, Arch. Rational Mech. Anal., 1980, 73: 69-77.

[30] J. H. Van Vuuren, The existence of travelling plane waves in a general class of competition-diffusion systems, IMA J. Appl. Math., 1995, 55: 135-148.

[31] Z. H. Zhao, R. Li, X. K. Zhao and Z. S. Feng, Traveling wave solutions of a nonlocal dispersal predator-prey model with spatiotemporal delay, Z. Angew. Math. Phys. 69 (2018), no. 6, Paper No. 146, 20 pp.

[32] W. J. Zuo and J. P. Shi, Traveling wave solutions of a diffusive ratio-dependent Holling-Tanner system with distributed delay, Comm. pure Appl. Anal., 2018, 17: 1179-1200. 